\title{
Pseudomembranous colitis in a pregnant woman
}

\author{
Mridula T, ${ }^{1}$ Pai RR, ${ }^{2}$ Mathai AM, ${ }^{3}$ Tantry BV, ${ }^{4}$ Adhikari $\mathrm{P}^{5}$
}

\author{
1-3 Department of Pathology, \\ ${ }^{4}$ Department of Gastroenterology, \\ ${ }^{5}$ Department of Gastroenterology, \\ Kasturba Medical College, Karnataka, India
}

\section{Corresponding author}

Dr Alka Mary Mathai

Department of Pathology

Kasturba Medical College, Karnataka, India.

alka977@gmail.com

\begin{abstract}
Pseudomembranous colitis in association with pregnancy has not been well described in English literature. Recent studies show a drastic increase in the incidence and severity of Pseudomembranous colitis in pregnant women, who were once thought to be at low risk. We report here a case of Pseudomembranous colitis in a young healthy immunocompetent pregnant lady. An early suspicion of this entity with the characteristic appearance of pseudomembranes on colonoscopy and histology confirmed the diagnosis enabling prompt treatment and complete recovery without any serious consequences.
\end{abstract}

Key Words

immunocompetent, pregnancy, pseudomembranous colitis

\section{INTRODUCTION}

Pseudomembranous colitis (PMC), most commonlycaused by Clostridium difficile, usually affects older individuals and hospitalized patients with chronic underlying disease or with recent antimicrobial therapy. ${ }^{1 .}$ There has been a recent increase in the incidence and severity of the infection worldwide because of the increased use of broad spectrum antibiotics and the emergence of a hypervirulent strain of the organism. ${ }^{2-4}$ Pregnant women were previously thought to be at low risk or developed mild disease due to their young age and overall good health. ${ }^{2,5}$ We herein describe a young healthy immunocompetent pregnant woman with infectious diarrhoea, diagnosed as PMC based on the endoscopic and histopathological features.

\section{CASE REPORT}

A 25 year old female at 25 weeks of gestation (gravida 2, para 1, living 1) presented with complaints of loose stools (4-6 times/day) and pain abdomen of twenty days duration. The abdominal pain would subside after passing stools. The patient had received roxithromycin for suspected urinary tract infection.
On physical examination, she was slightly febrile. Her vital signs were stable. On abdominal examination, uterus was 24-26 weeks size and bowel sounds were present. There was no abdominal tenderness. All other systems were within normal limits. A clinical diagnosis of infectious diarrhoea was made and oral ampicillin was advised. The diarrhoea aggravated with mucus and streaks of blood in stool. Laboratory investigations showed a white blood cell count of 15,000 cells $/ \mathrm{mm}^{3}$ with neutrophilia. Stool examination showed 1-2 pus cells/ high power field and was positive for occult blood. Stool culture did not isolate any intestinal pathogens. Cytotoxicity test for Clostridium difficile infection was not available. Hence, a colonoscopy was done. Colonoscopy revealed discrete ulcers of varying sizes covered with yellowish pseudomembranes in the rectum. The sigmoid colon was normal. Biopsy was performed. Histopathologically, colonic mucosa showed necrosis of superficial portion of colonic crypts with an inflammatory pseudomembrane made up of fibrin and neutrophils mushrooming from degenerative crypts consistent with PMC (Figure 1). Underlying mucosa showed acute over chronic inflammatory cell infiltrate with cryptitis. Goblet cell depletion was seen. She was then started on oral metronidazole $400 \mathrm{mg}$ three times daily to which she responded and her symptoms subsided. She had completely recovered on reviewing after ten days. 


\section{DISCUSSION}

PMC, most commonly due to Clostridium difficile infection, is an entity described before the antibiotic era. ${ }^{6}$ Although pregnant women were once thought to be at low risk, severe Clostridium difficile infection with higher mortality, foetal loss and morbidity requiring colectomy have been described recently in peripartum women., ${ }^{2,7}$ The proposed underlying mechanism include the suppressed pro-inflammatory cytokine production and cell-mediated immunity resulting from the elevated cortisol, oestrogen and progesterone hormones which themselves have antiinflammatory effects at high levels during the later half of pregnancy, ${ }^{2,8}$ Hence the protective role of host anti-toxin A IgG against C. difficile is bypassed in severe cases. A higher carrier rate in the female urogenital tract is also described as contributory factor. There have been reported cases of PMC following caesarean section due to use of prophylactic antibiotics. ${ }^{3,5,9}$

Clostridium difficile, a Gram-positive, anaerobic, spore forming bacilli causes exogenous infection by spores that are acquired by environmental contamination. ${ }^{10}$ It colonizes the colonic mucosa of individuals in whom the normal bacterial flora is altered by administration of antibiotics. This results in overgrowth of $C$. difficile that renders its action by exotoxins causing mucosal damage and inflammation resulting in diarrhoea and colitis. ingDose, route and duration of administration of antibiotics is not important and does not directly affect causation of PMC. ${ }^{11}$

PMC is characterized by inflammatory plaques and pseudomembranes in the colonic mucosa. The clinical manifestation varies from asymptomatic carriage or mild diarrhoea without pseudomembrane formation to PMC and severe fulminant fatal colitis mimicking ulcerative colitis. The mucosal oedema of the colon may appear as thumbprinting on abdominal scan or as thickened colonic mucosa on computed tomography scan. The complications include toxic megacolon, ascites, perforation, prolonged ileus and even death. The diarrhoea often subsides when the antibiotics is stopped, in which case specific treatment in not necessary.

The laboratory diagnosis of $\mathrm{C}$. difficile infection with or without PMC is based on either isolation of the organism by stool culture or by cytotoxin assay, detecting C. difficle cytotoxin in stool which has high sensitivity of $94 \%$ to $100 \%$ and specificity of $99 \%{ }^{3}$ In case of failure to establish a diagnosis, imaging modalities and colonoscopy is indicated. The presence of typical psuedomembranes in the form of multiple adherent yellow plaques of varying size on the colonic mucosa with normal or mild hyperaemic surrounding mucosa is pathognomonic of PMC and is a strong evidence for immediate treatment. ${ }^{12}$

It is effectively treated with oral vancomycin or metronidazole. Newer therapeutic modalities include, new antimicrobial agents, toxin-binding products, and immunization, however, their safety in pregnancy is controversial. $^{2}$ Early recognition and close monitoring of the signs and symptoms is essential during pregnancy. Increase in abdominal tenderness, blood leukocyte counts and serum creatinine levels suggest deterioration and impending complications. ${ }^{3}$ In case of severe disease complicated with ileus or toxic megacolon, treatment varies from delivery of vancomycin to diseased areas via nasogastric or rectal tubing to life saving subtotal colectomy.

In the present case, use of antibiotics (roxithromycin followed by ampicillin) for suspected urinary tract infection and infective diarrhoea predisposed to PMC in a pregnant lady. The characteristic colonoscopic appearance and histopathologic analysis enabled a proper diagnosis and prompt treatment with complete recovery.

To conclude, PMC or antibiotic associated diarrhoea should be suspected in patients who develop low grade fever, diarrhoeal or abdominal symptoms during or following antibiotic exposure. An early diagnosis and prompt treatment can avoid a fulminant disease and its consequences and also the morbidity and mortality associated with unnecessary surgical interventions. This case report hopefully will increase the awareness needed regarding the growing incidence of $\mathrm{C}$. difficle- associated diarrhoea in obstetric women where antibiotics have to be judiciously used and warranted only if there is a definite indication.

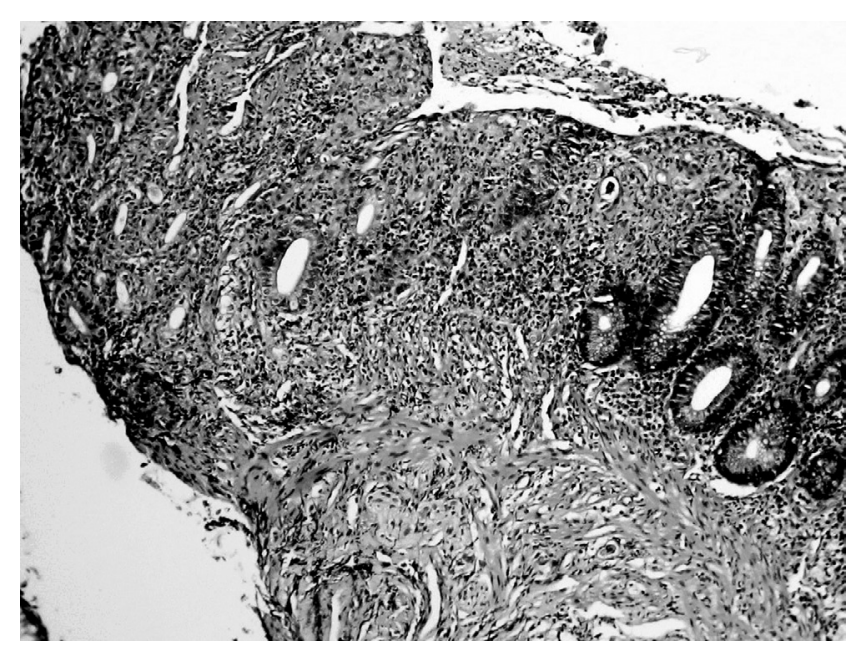

Figure 1. Ulcerated colonic mucosa covered by inflammatory pseudomembrane made up of fibrin and neutrophils (Haematoxylin and eosin $\times 100$ ) 


\section{REFERENCES}

1. Asha NJ, Tompkins D, Wilcox MH. Comparative analysis of prevalence, risk factors, and molecular epidemiology of antibiotic-associated diarrhea due to Clostridium difficile, Clostridium perfringens, and Staphylococcus aureus. J Clin Microbiol. 2006;44:2785-91.

2. Rouphael NG, O’Donnell JA, Bhatnagar J, Lewis F, Polgreen PM, Beekmann S, et al. Clostridium difficile-associated diarrhea: an emerging threat to pregnant women. Am J Obstet Gynecol. 2008;198:635.e1-6.

3. Ghai S, Ghai V, Sunderji S. Fulminant postcesarean Clostridium difficile pseudomembranous colitis. Obstet Gynecol. 2007;109(2 Pt2):541-3.

4. Pépin J, Valiquette L, Alary ME, Villemure P, Pelletier A, Forget $\mathrm{K}$, et al. Clostridium difficile-associated diarrhea in a region of Quebec from to 2003: a changing pattern of disease severity. Can Med Assoc J. 2004; 171:466-72.

5. James AH, Katz VL, Dotters DJ, Rogers RG. Clostridium difficile infection in obstetric and gynecologic patients. South Med J. 1997;90:889-92.

6. Pothoulakis C, LaMont JT. Clostridium difficile colitis and diarrhea. In: Giannella RA, editor. Acute infectious diarrhea. Gastroenterology Clinics of North America Vol 22 No.3 USA: W.B. Saunders Co; 1993. p.623 - 37.
7. Centers for Disease Control and Prevention (CDC). Severe Clostridium difficile-associated disease in populations previously at low risk--four states, 2005. MMWR Morb Mortal Wkly Rep. 2005;54:1201-5

8. Calcagni E, Elenkov I. Stress system activity, innate and T helper cytokines, and susceptibility to immune-related diseases. Ann N Y Acad Sci. 2006; 1069:62-76. .

9. Arsura EL, Fazio RA, Wickremesinghe PC. Pseudomembranous colitis following prophylactic antibiotic use in primary cesarean section. Am J Obstet Gynecol. 1985;151:87-9.

10. Garey KW, Jiang ZD, Yadav Y, Mullins B, Wong K, Dupont HL. Peripartum Clostridium difficile infection: case series and review of the literature. Am J Obstet Gynecol. 2008; 199:332-7

11. Griffiths JK, Gorbach SL. Other bacterial diarrhoeas. Baillieres Clin Gastroenterol. 1993;7:263-305.

12. Kelly CP, Pothoulakis C, LaMont JT. Clostridium difficile colitis. N Engl J Med. 1994;330:257-62. 